\title{
The Coupling Effect on Torsional and Longitudinal Vibrations of Marine Propulsion Shaft System
}

\author{
๑ (1) Akile Neşe Halilbeşe ${ }^{1}$, (1) Osman Azmi Özsoysal ${ }^{2}$ \\ ${ }^{1}$ Ordu University, Department of Marine Engineering, Ordu, Turkey \\ ${ }^{2}$ İstanbul Technical University, Department of Naval Architecture and Marine Engineering, İstanbul, Turkey
}

\begin{abstract}
The propulsion shaft system is one of the essential parts of the ships due to its reliability and stabilization directly affecting the safety in operation. The propulsion system transmits the torque generated by the engine to the propeller via the main shaft. During its navigation, torsional, longitudinal, and transversal vibrations inevitably occur, and precautions must be taken during the design stage to prevent system damage and reduce power transmission efficiency. In this article, three dissimilar models numerically generated by the lumpedmass method are used to investigate the harmonic conclusions of forced coupled torsional and longitudinal vibrations of the system. Numerical results correlated with the experimental results at rotational speed and load acting onto the propulsion shaft system. A further finding is to create a third method upon discussing the facts revealed by analyzing the advantages and disadvantages of the models, especially considering differences between the first two models.
\end{abstract}

Keywords

Coupled torsional-longitudinal vibrations, Forced vibrations, Marine propulsion shaft system, Lumped-mass method

\section{Introduction}

The reliability of a cruising vessel is directly related to the safety of the propeller-shaft system. Ships are subjected to different forces during navigation, such as waves, wind, and other exciting forces [1]. These forces create extreme vibrations in torsional, longitudinal, and transverse modes and their coupled forms, resulting in an undesirable and intensified vibration response of the shaft [2]. Coupled vibrations would raise the noise level by stimulating the shafts and foundation, bearings, and hull. As a result, the poor performance of the system will lead to fatigue, fracture, and tribological problems on the shaft, and even failures for the overall shaft system. Consequently, the ship's navigation reliability will be significantly reduced [3].

The majority of the studies on the dynamic behavior of the propulsion system deals with the single-axis vibrations by ignoring the coupled vibrations, resulting in different results by the actual measurements [4,5]. Torsional vibration is the foremost dangerous type for the crankshaft, bearings, and shaft lining and is mostly studied in singleaxis vibration investigations [6]. Huang and Horng [7] used the transfer matrix method for torsional vibration analysis of damped systems. Wu and Yang [8] studied free and forced torsional vibration problems for the multidegree of freedom system using the transfer matrix method. The finite element method was frequently applied for torsional vibration analysis [9-11]. Besides, some of these studies on torsional, longitudinal, and transverse vibration were evaluated by experiments [12,13]. Gan-boa and Yaoa [14] used the reduced-order modeling method to control the longitudinal vibration of a marine propulsion shaft system. Earlier research focused on separate torsional and longitudinal vibrations without interaction between them. It is observed that eliminating the interaction significantly distorts results for the measurements [15]. Huang et al. [16] also reported that only a few studies in the literature that focus on the coupled vibrations of propeller-shaft systems.

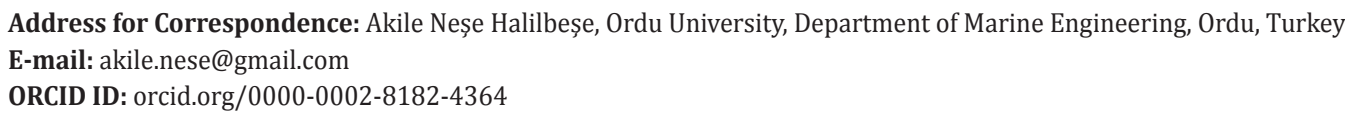

To cite this article: A. N. Halilbeşe, and O. A. Özsoysal, “The Coupling Effect on Torsional and Longitudinal Vibrations of Marine Propulsion Shaft System.” Journal of ETA Maritime Science, vol. 9(4), pp. 274-282, 2021.

${ }^{\circ}$ Copyright 2021 by the Journal of ETA Maritime Science published by UCTEA Chamber of Marine Engineers 
Parsons [17] first showed the coupled torsional and longitudinal vibrations of propeller-shaft systems, considering the coupling through added mass and hydrodynamic damping coefficients due to the propeller. The Ritz method and Lagrange equation, as well as Hamilton's principle discretized by the Galerkin method, are used to investigate coupled longitudinal-transverse vibrations of a propulsion shaft system $[18,19]$. Using the lumped-mass method, Huang et al. [16] studied the coupled torsionallongitudinal problem experimentally and numerically. Unlike the studies that considered the coupling through added mass and hydrodynamic damping coefficients, they examined the system's response by considering the coupling through a stiffness matrix using specific values to this coefficient. Moreover, the longitudinal vibration of the system was predicted by ignoring the bearings, and a different path was considered by splitting the shaft into masses compared to other publications on this topic.

In this study, the first model proposed by Huang et al. [16] and the second model obtained the coupling effect from the propeller are compared for the coupled torsionallongitudinal vibrations of a propeller-shaft system. To more accurately observe the effect of differences between Model 1 and Model 2 on the coupled vibrations, a new model, Model 3 , is formed by utilizing these differences. Experimental verifications are also provided for those three models. The lumped-mass method is adopted for both coupled and uncoupled torsional-longitudinal vibration. The response amplitudes due to harmonic loadings are presented to observe the effect of coupling.

\section{Methodology and Numerical Model}

In this study, the coupled torsional-longitudinal vibrations of a propeller-shaft system are numerically studied using the lumped-mass method and adopting the approaches described as Model 1 and Model 2, respectively. After evaluating the differences between these two models, a new analysis model was decided, and Model 3 was formed.

The shaft can be modeled as an Euler beam with a mass payload, equivalent to a cantilever beam with its mass center away from the centerline [20,21]. The shaft is modeled using the Euler beam, with the engine as the fixed end and the propeller as the free end $[16,22]$. Figure 1 presents the installation diagram, consisting of four different parts. The gravity center of the attached mass, i.e., propeller, may not

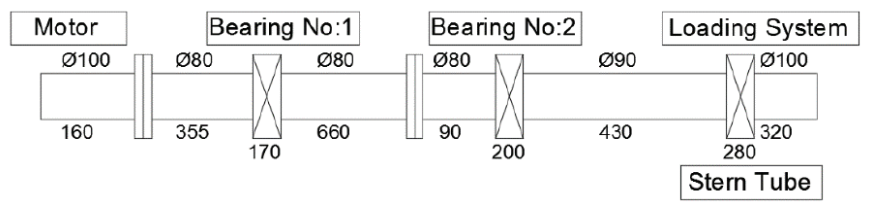

Figure 1. Details of the shaft system coincide with its attachment point to the beam. This beam can generally be subjected to torsional and longitudinal deformations due to the acting forces $[16,23]$.

When the propeller starts to rotate and then experiences longitudinal or torsional vibrations, it will develop an oscillatory lift that produces both an axial force and a torque motion [17]. Considering the uncoupled torsional and longitudinal vibrations, the equation of motion of the shaft system, in the discretized form, can be given as (Equation 1 is below):

$$
\begin{aligned}
& \boldsymbol{J} \ddot{\theta}+\boldsymbol{D}_{t} \dot{\theta}+\boldsymbol{K}_{t} \theta=T \sin (\omega t) \\
& \boldsymbol{M} \ddot{x}+\boldsymbol{D}_{\boldsymbol{x}} \dot{x}+\boldsymbol{K}_{x} x=F \sin (\omega t)
\end{aligned}
$$

Here, $\boldsymbol{M}$ is the mass matrix; $\boldsymbol{J}$ is the moment of inertia; $\boldsymbol{K}_{\boldsymbol{t}}$ and $\boldsymbol{K}_{x}$ represent the torsional stiffness and longitudinal stiffness, respectively; $\boldsymbol{D}_{\boldsymbol{t}}$ and $\boldsymbol{D}_{\boldsymbol{x}}$ represent the torsional damping and longitudinal damping, respectively; $T$ and $F$ represent the amplitudes of the torque and axial force induced by the propeller, respectively; $\boldsymbol{\theta}$ and $\boldsymbol{x}$ denote the torsional angle and axial displacement, respectively; $w$ is the excitation frequency. Since there is no thrust bearing in the experimental setup, the entire shaft system is modeled for calculating longitudinal vibrations. Figure 2 shows the experimental setup, including disks attached to the shaft instead of crankshafts. While there is no combustion in the experimental setup, the torque caused by the gas pressure does not exist. Besides, the inertia and torque of the disks are quite small compared to the shaft; consequently, disks are not included in the numerical model.

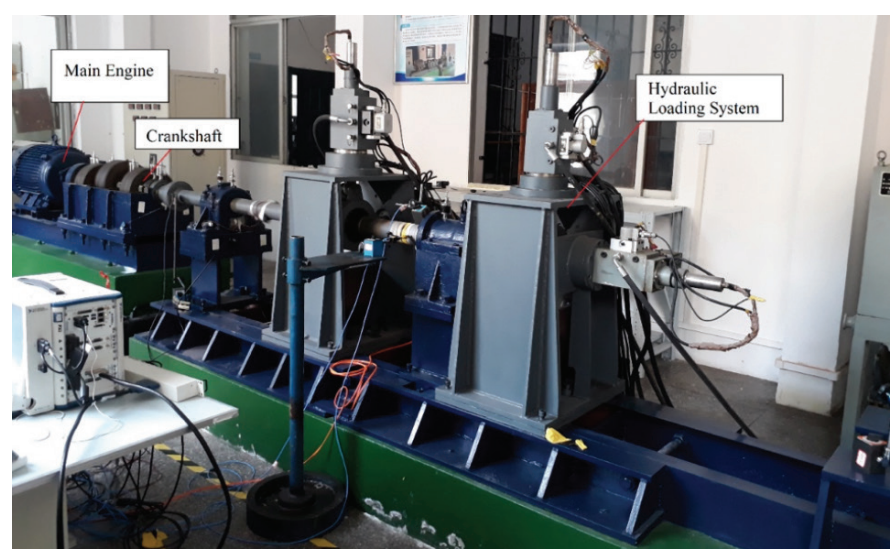

Figure 2. Experimental setup of the propulsion shaft system

The equation of the motion of the marine propulsion shaft system is shown for the undamped and unforced system with Equation 2 to define the coupling effect. When the propeller rotates and vibrates either longitudinally or torsionally, it will develop an oscillatory lift that will produce both an oscillatory axial force and oscillatory torque on the propeller. These components will provide the coupled 
longitudinal and torsional motion. Neglecting the damping that occurred on the system, the hydrodynamic force and moment proportional to the acceleration will produce the following coupled equations of motion [17];

For the four sectioned shaft system, the moment of inertia and mass matrices are given as follows:

$J=\left[\begin{array}{cccc}J_{1} & 0 & 0 & 0 \\ 0 & J_{2} & 0 & 0 \\ 0 & 0 & J_{3} & 0 \\ 0 & 0 & 0 & J_{4}\end{array}\right], M=\left[\begin{array}{cccc}m_{1} & 0 & 0 & 0 \\ 0 & m_{2} & 0 & 0 \\ 0 & 0 & m_{3} & 0 \\ 0 & 0 & 0 & m_{4}\end{array}\right]$,

the torsional damping and longitudinal damping matrices are defined as follows:

$D_{t}=\left[\begin{array}{cccc}d_{t_{1}} & -d_{t_{1}} & 0 & 0 \\ -d_{t_{1}} & d_{t_{1}}+d_{t_{2}} & -d_{t_{2}} & 0 \\ 0 & -d_{t_{2}} & d_{t_{2}}+d_{t_{3}} & -d_{t_{3}} \\ 0 & 0 & -d_{t_{3}} & d_{t_{3}}\end{array}\right]$

$D_{x}=\left[\begin{array}{cccc}d_{x_{1}} & -d_{x_{1}} & 0 & 0 \\ -d_{x_{1}} & d_{x_{1}}+d_{x_{2}} & -d_{x_{2}} & 0 \\ 0 & -d_{x_{2}} & d_{x_{2}}+d_{x_{3}} & -d_{x_{3}} \\ 0 & 0 & -d_{x_{3}} & d_{x_{3}}\end{array}\right]$

and the torsional stiffness matrix is defined as follows:

$$
K_{t}=\left[\begin{array}{cccc}
k_{t_{1}} & -k_{t_{1}} & 0 & 0 \\
-k_{t_{1}} & k_{t_{1}}+k_{t_{2}} & -k_{t_{2}} & 0 \\
0 & -k_{t_{2}} & k_{t_{2^{2}}}+k_{t_{3}} & -k_{t_{3}} \\
0 & 0 & -k_{t_{3}} & k_{t_{3}}
\end{array}\right]
$$

Here, the coefficients $d_{t}$ and $d_{x^{\prime}}$ defining the torsional and longitudinal damping for the $j$ th shaft, are given by $d_{t_{j}}=0.08 J_{j} \omega_{j}, \quad d_{x_{i}}=0.05 m_{j} \omega_{j}, \quad$ respectively, and the coefficients $k_{t}$ defining the torsional stiffness is given by $k_{t}=G J_{j} / L_{j}$ in which $L_{j}$ is the length of the $j$ th section [16]. Since the longitudinal stiffness depends on the selected solution model, it can be presented as follows:

The external forcing in Equation (1) can be defined as

$$
T=\left[\begin{array}{llll}
T_{t} & 0 & 0 & 0
\end{array}\right] F=\left[\begin{array}{llll}
F_{x} & 0 & 0 & 0
\end{array}\right]
$$

and the response of the system is represented as follows:

$$
\theta=\left[\begin{array}{llll}
\theta_{1} & \theta_{2} & \theta_{3} & \theta_{4}
\end{array}\right] x=\left[\begin{array}{llll}
x_{1} & x_{2} & x_{3} & x_{4}
\end{array}\right]
$$

The proposed solution models for the coupled torsionallongitudinal vibration of the propeller-shaft system differ from each other depending on the adopted coupling mechanism, as briefly discussed next.

\section{Model 1}

The coupled vibrations of the system are described as [21] follows:

$$
\left[\begin{array}{cc}
M & 0 \\
0 & J
\end{array}\right]\left[\begin{array}{c}
\ddot{x} \\
\ddot{\theta}
\end{array}\right]+\left[\begin{array}{cc}
D_{x} & 0 \\
0 & D_{t}
\end{array}\right]\left[\begin{array}{c}
\dot{x} \\
\dot{\theta}
\end{array}\right]+\left[\begin{array}{cc}
K_{x} & -K_{x t} \\
-K_{t x} & K_{t}
\end{array}\right]\left[\begin{array}{l}
x \\
\theta
\end{array}\right]=\left[\begin{array}{l}
F \\
T
\end{array}\right]
$$

Here, the longitudinal stiffness is given by

$$
K_{x}=\left[\begin{array}{cccc}
k_{x_{1}} & -k_{x_{1}} & 0 & 0 \\
-k_{x_{1}} & k_{x_{1}}+k_{x_{2}} & -k_{x_{2}} & 0 \\
0 & -k_{x_{2}} & k_{x_{2}}+k_{x_{3}} & -k_{x_{3}} \\
0 & 0 & -k_{x_{3}} & k_{x_{3}}+k_{x_{4}}
\end{array}\right]
$$

Where $k_{x_{i}}=E A / L_{j}$; the coupling between axial and rotational motions is introduced through the diagonal stiffness matrices $K_{t x^{\prime}} K_{x t^{\prime}}$ which are defined by $k_{x t_{j}}=\delta k_{t^{\prime}} k_{t x_{j}}=\delta k_{x j^{\prime}}$ The equation of the motion for the free vibrations of the marine propulsion shaft system can be given to define the coupling effect, as follows:

$\left[\begin{array}{cc}m & m_{c} \\ m_{c} & J\end{array}\right]\left[\begin{array}{c}\ddot{x} \\ \ddot{\theta}\end{array}\right]+\left[\begin{array}{cc}k_{x} & 0 \\ 0 & k_{t}\end{array}\right]\left[\begin{array}{l}x \\ \theta\end{array}\right]=0$

Here,mintroduces inertial coupling depending on the propeller characteristics. Using the solutions $x=\operatorname{ReX} e^{i \omega t}$ and $\theta=\operatorname{Re} \Theta e^{i \omega t}$ in Equation (2), the following eigenvalue problem is obtained:

$\left[\begin{array}{cc}\left(k_{x}-m \omega^{2}\right) & -m_{c} \omega^{2} \\ -m_{c} \omega^{2} & \left(k_{\theta}-J \omega^{2}\right)\end{array}\right]\left[\begin{array}{l}x \\ \theta\end{array}\right]=0$

By defining $\omega_{x}=\sqrt{k_{x} / m}$ and $\omega_{t}=\sqrt{k_{t} / m}$, the characteristic equation for Equation (4) can be given as follows:

$$
\omega^{4}(1-\delta)-\omega^{2}\left(\omega_{t}^{2}+\omega_{x}^{2}\right)+\omega_{x}^{2} \omega_{t}^{2}=0
$$

Here the coupling coefficient $\delta$, which describes the longitudinal deformation induced by the torsional angle, is defined as

$$
\delta=\frac{m_{c}^{2}}{m J}
$$

Due to a lack of propeller in the experimental setup, this study adopts 0.02 value from the literature $[17,20]$.

\section{Model 2}

In Model 2, each shaft is divided into two masses for longitudinal vibration, and the moment of inertia of the 
shaft is neglected considering that it is relatively small compared to the corresponding values of the propeller and gears, including two components that are omitted in Model 1. For presenting a well-suited assessment of the models compared with the test results in Section 4, each shaft is modeled as a mass, and moments of inertia of the shafts are inevitably taken into account as the test system involves no propeller or gears. Unlike Model 1, the torsional and longitudinal vibrations are coupled due to the radiated flow from the propeller in Model 2, and the interaction terms are given in terms of fluid added mass $\left(M_{c}\right)$, hydrodynamic damping $\left(D_{c}\right)$ and hydrodynamic stiffness $\left(K_{c}\right)$. The equation of motion of the shaft system is as follows:

$$
\left[\begin{array}{cc}
M & M_{c}^{T} \\
M_{c} & J
\end{array}\right]\left[\begin{array}{c}
\ddot{x} \\
\ddot{\theta}
\end{array}\right]+\left[\begin{array}{cc}
D_{x} & D_{c}^{T} \\
D_{c} & D_{t}
\end{array}\right]\left[\begin{array}{c}
\dot{x} \\
\dot{\theta}
\end{array}\right]+\left[\begin{array}{cc}
K_{x} & K_{c}^{T} \\
K_{c} & K_{t}
\end{array}\right]\left[\begin{array}{c}
x \\
\theta
\end{array}\right]=\left[\begin{array}{l}
F \\
T
\end{array}\right]
$$

Hence, the interaction terms for the system given in Figure 1 are zero due to the lack of propeller; the resulting equation of motion describing the uncoupled vibrations of the propeller-shaft system can be defined as follows:

$$
\left[\begin{array}{cc}
M & 0 \\
0 & J
\end{array}\right]\left[\begin{array}{l}
\ddot{x} \\
\ddot{\theta}
\end{array}\right]+\left[\begin{array}{cc}
D_{x} & 0 \\
0 & D_{t}
\end{array}\right]\left[\begin{array}{l}
\dot{x} \\
\dot{\theta}
\end{array}\right]+\left[\begin{array}{cc}
K_{x} & 0 \\
0 & K_{t}
\end{array}\right]\left[\begin{array}{l}
x \\
\theta
\end{array}\right]=\left[\begin{array}{l}
F \\
T
\end{array}\right]
$$

Since bearings are allowed in the system and coupling has no stiffness component, the longitudinal stiffness matrix is different from Model 1 as follows:

$$
K_{x}=\left[\begin{array}{cccc}
k_{x_{1}} & -k_{x_{1}} & 0 & 0 \\
-k_{x_{1}} & k_{x_{1}}+k_{x_{2}}+k_{x_{5}} & -k_{x_{2}} & 0 \\
0 & -k_{x_{2}} & k_{x_{2}}+k_{x_{3}}+k_{x_{6}} & -k_{x_{3}} \\
0 & 0 & -k_{x_{3}} & k_{x_{3}}+k_{x_{4}}+k_{x_{7}}
\end{array}\right]
$$

Here, $k_{x_{j}}=E A / L_{j} \quad k_{x_{s}}, k_{x_{6}}, k_{x_{j}}$ represent the stiffness of the bearings, considering that they are attached to the foundation, which is attached to the ground, and their values are considered as $1.0 \mathrm{GN} / \mathrm{m}[24,25]$. Figure 1 shows the location of the bearings, which are included in the mass elastic system from the connection points.

\section{Model 3}

Model 3 is formed by collecting the coupling mechanism inherent to Model 1 and the bearing mechanism in Model 2 to provide more realistic predictions of the coupled dynamic behavior of the propeller-shaft system-it involves inertia, damping, and stiffness coupling terms. The coupled torsional-longitudinal vibrations of the system are now defined by

$$
\left[\begin{array}{cc}
M & 0 \\
0 & J
\end{array}\right]\left[\begin{array}{l}
\ddot{x} \\
\ddot{\theta}
\end{array}\right]+\left[\begin{array}{cc}
D_{x} & 0 \\
0 & D_{t}
\end{array}\right]\left[\begin{array}{l}
\dot{x} \\
\dot{\theta}
\end{array}\right]+\left[\begin{array}{cc}
K_{x} & -K_{x t} \\
-K_{t x} & K_{t}
\end{array}\right]\left[\begin{array}{l}
x \\
\theta
\end{array}\right]=\left[\begin{array}{l}
F \\
T
\end{array}\right]
$$

All entities in Equation (16) are previously defined; the mass moment of inertia matrix $(J)$ and mass matrix $(M)$ are given in Equation (6). The torsional and longitudinal stiffness matrices of the system are given in Equation (8), and Equation (15) whereas torsional and longitudinal damping matrices are given as Equation (7a) and Equation (7b). The stiffness matrices $K_{t x}$ and $K_{x t}$ are defined by $\delta \times K_{\chi} K_{t}$

\section{Experimental Setup}

The coupled torsional-longitudinal vibration of the shaft system is also experimentally investigated to verify the numerical predictions. The general view of the test bed and its installation are shown in Figure 2. The experimental setup consists of an actuator that represents the propeller connected to the end of the shaft, a bearing for preventing hogging or sagging of the unsupported span of the shaft, a drive unit acting as a marine engine at the other end of the shaft, a foundation for mounting the whole plants, and a base frequency converter.

The propulsion shaft system consists of two intermediate shafts and a tail shaft. The shafts are connected using a hydraulic coupling. Two intermediate bearings support the intermediate shafts, and another stern bearing supports the tail shaft [5]. Theoretical material properties are considered as $E=206 \mathrm{GPa}, G=77 \mathrm{GPa}$, and $p=7850 \mathrm{~kg} / \mathrm{m}^{3}$. Table 1 presents the geometrical properties of each shaft segment, where the order is from the fixed end (engine side) to the free end (propeller side). Figure 3 shows measurement points by locating the sensors transmitting the analog signals.

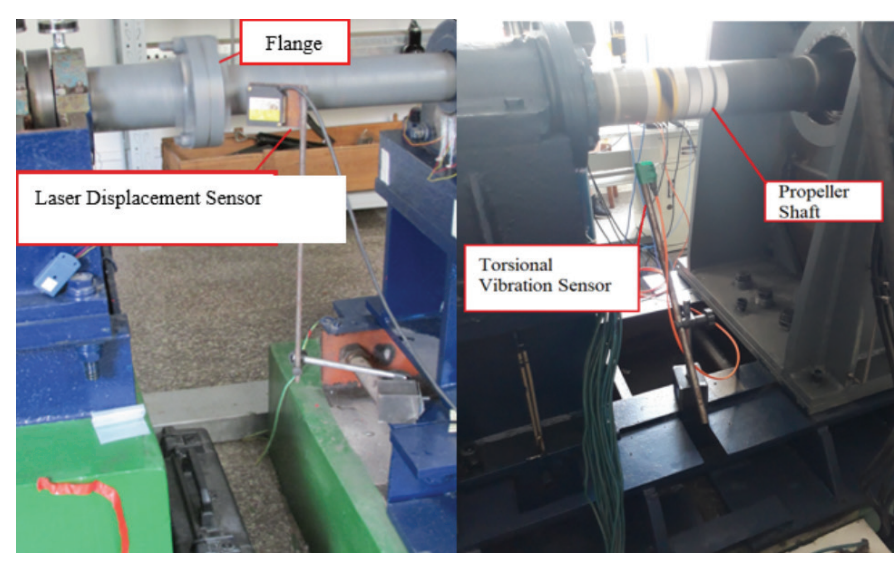

Figure 3. Measurement points layout for the shaft longitudinal and torsional vibrations

Table 1. Geometrical properties of the shaft segments

\begin{tabular}{|c|c|c|c|c|}
\hline Shaft no & $\mathbf{1}$ & $\mathbf{2}$ & $\mathbf{3}$ & $\mathbf{4}$ \\
\hline Length (m) & 0.6 & 0.935 & 0.67 & 0.46 \\
\hline Diameters (m) & 0.08 & 0.08 & 0.09 & 0.1 \\
\hline
\end{tabular}


Both torsional and longitudinal vibration signals are recorded simultaneously along the shaft. Signals were recorded at $0.0005 \mathrm{~s}$ intervals for $1 \mathrm{~min}$. The laser torsional vibration meter (B\&K MM0071 sensor and 2523 laser) is used to measure the signal of the tail shaft and to check and compare with the shaft speed, as shown in Figure 4. The laser's measurement frequency range is 0.5 to 3000 $\mathrm{Hz}$, and its sensitivity is $100 \mathrm{mV} / \mathrm{g}$. The flange surface at the intermediate shaft is selected for measuring the longitudinal vibration, where the laser displacement sensor (OPTEX CD33) is used. The sensor position is fixed to reduce possible errors due to the vibration. Longitudinal loads are adjusted using a hydraulic system. Repeat accuracy is also $2 \mu \mathrm{m}^{*}$, and high-accuracy displacement measurement is supported. The torque generated by the longitudinal force is measured using a strain gage placed on the intermediate shaft. Shaft speed is increased from $100 \mathrm{rpm}$ to $190 \mathrm{rpm}$ with an interval of $30 \mathrm{rpm}$, and displacement amplitudes for the shaft coupled vibrations are recorded for each speed. However, the measurements and results presented in Section 4 are only for $100 \mathrm{rpm}$. The amplitudes of the applied axial force and corresponding torque are 0.055 $\mathrm{Nm}$ and $86 \mathrm{kN}$, respectively, for $100 \mathrm{rpm}$. Since the test apparatus allows only axial and transversal loadings, the torsional stress could not be obtained directly, yet torque values are acquired from the measured longitudinal

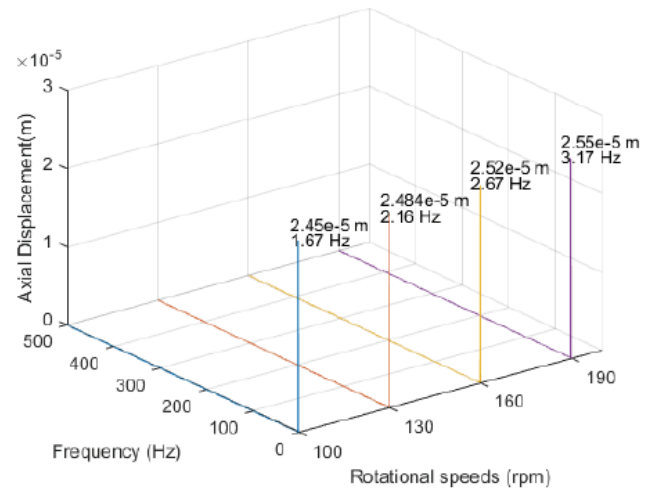

a)

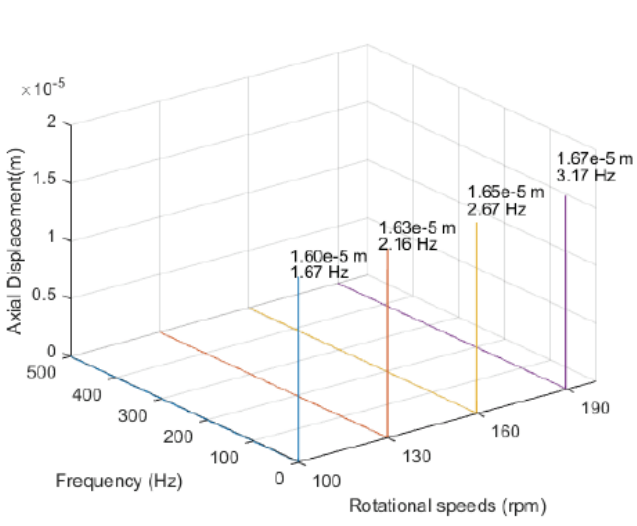

stresses. The longitudinal stresses are collected for the axial forces applied in terms of the displacements of the loading system. Table 2 presents applied displacements, measured torque, and axial force amplitudes.

Table 2. Torque and axial force values for the considered rotational speeds

\begin{tabular}{|c|c|c|c|c|}
\hline Rotational speed $(\mathrm{rpm})$ & 100 & 130 & 160 & 190 \\
\hline Displacement $(\mathrm{mm})$ & 0.6 & 0.9 & 1.2 & 1.5 \\
\hline Axial force $(\mathrm{kN})$ & 85.904 & 87.129 & 88.359 & 89.586 \\
\hline Torque $(\mathrm{Nm})$ & 0.055 & 0.125 & 0.275 & 0.325 \\
\hline
\end{tabular}

\section{Application and Discussion}

For achieving stable results, simulation time is taken as 10 seconds. The initial conditions are applied as $\theta_{j}=x_{j}=\dot{\theta}_{j}=\dot{x}_{j}=0$. The unknown coupling stiffness coefficients are assumed to be $k_{t x}=k_{x t}=0$.

\subsection{Verification}

Figure 5 and 6 indicate the axial displacement and torsional angle values obtained from all numerical models and experiments at 100-190 rpm shaft speed. The hydrodynamic effect is presented using the coupling stiffness coefficient in Method 1 [17]. The bearings are considered, and the coupled

b)
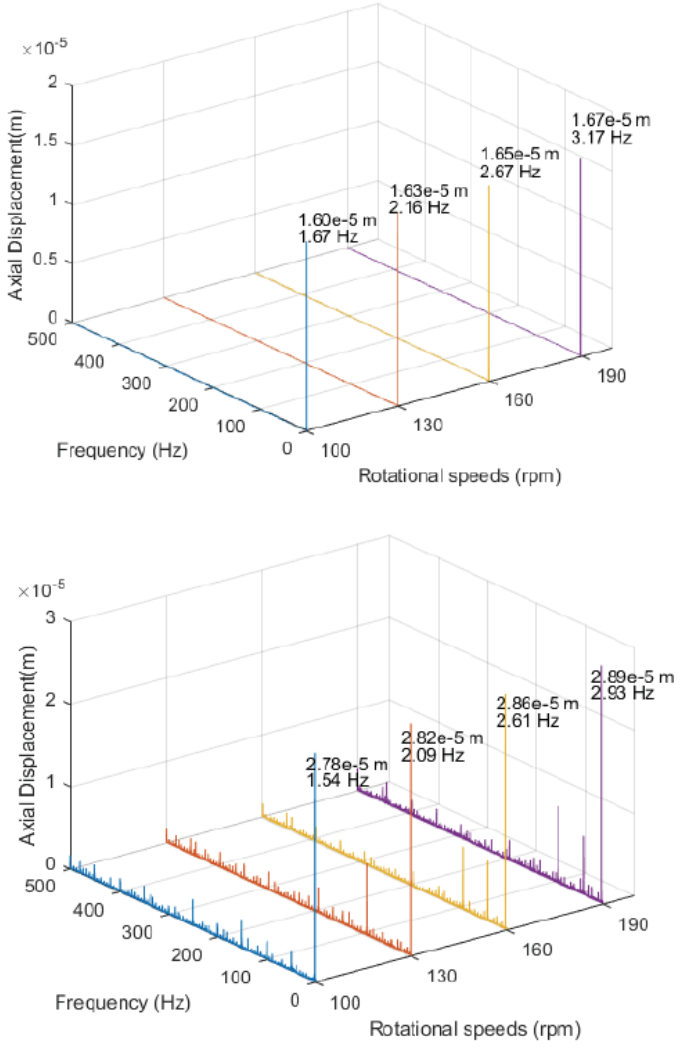

Figure 4. Axial displacement results at $\omega=100-190$ rpm. a) Model 1, b) Model 2, c) Model 3, d) Experiment 

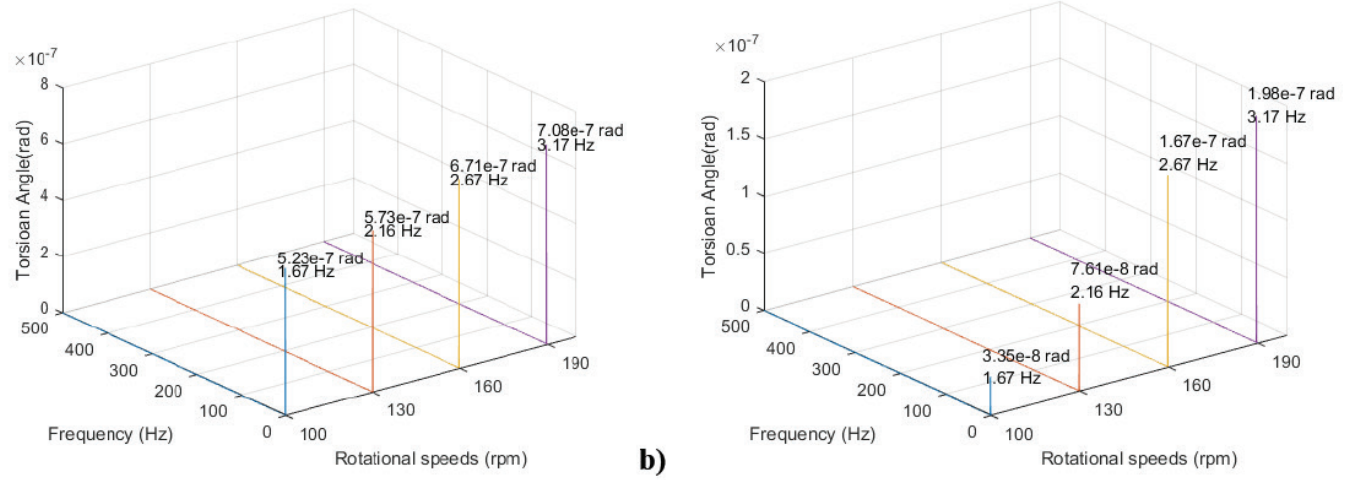

a)

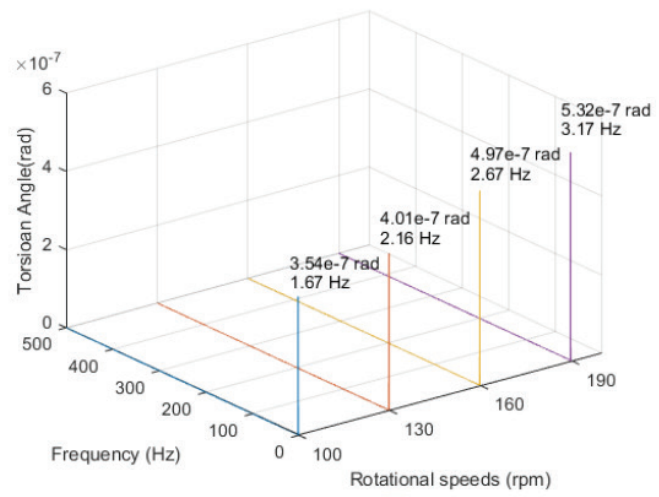

b)

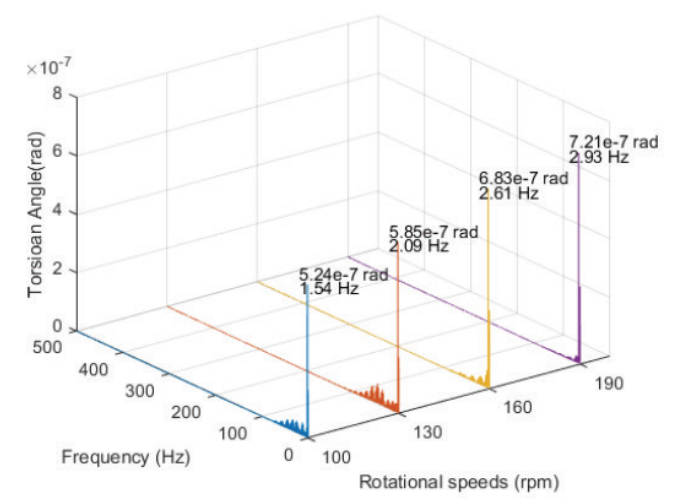

Figure 5. Torsional angle results at $\omega=100-190 \mathrm{rpm}$ a) Method 1, b) Method 2, c) Method 3, d) Experiment
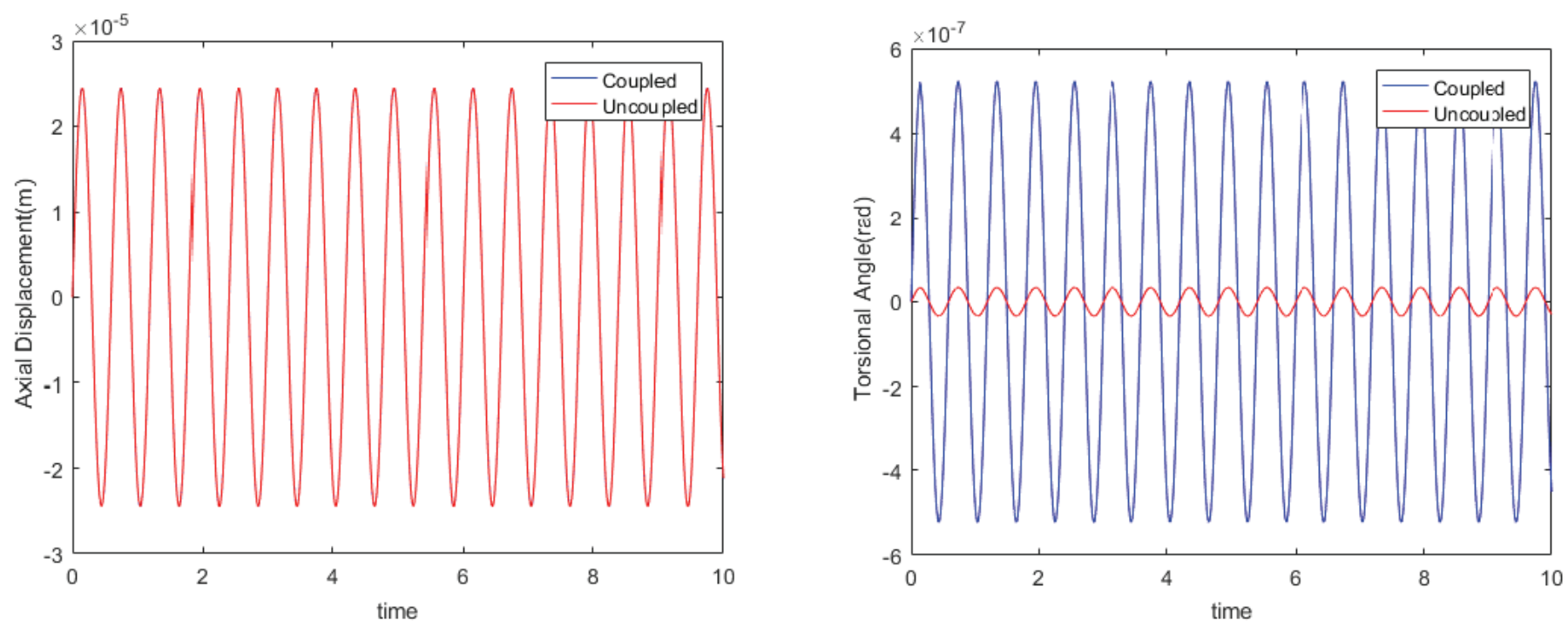

Figure 6. Axial displacements and torsional angles for Model 1 at $100 \mathrm{rpm}$

vibration effect could not be modeled by the hydrodynamic effects in Method 2 due to the lack of a propeller. Because of the inability to calculate hydrodynamic coefficients in Model 2, axial displacement and torsional angle are calculated for uncoupled vibration. Figure 4 indicates the alteration in axial vibration of experiment and numerical models by increasing rotational speed. Numerical and experimental results are given for a range of rotational speeds (100-190 rpm) to find out if they match well with each other; also, the similarity of error margins for different speed values increased the reliability of the experiment. Figure $52.78 \times 10^{-5} \mathrm{~m}$ for the experiment at $100 \mathrm{rpm}$ rotational speed, respectively. As might be expected, the test result is higher than numerical predictions owing to the imperfection of theoretical models, 
but the margin of error is low and numerical methods are very much compatible with experiment results. The results obtained from Model 2 and Model 3 show that the coupled vibration for each rotational speed value has virtually no effect on axial displacement. By comparing Model 1 and Model 3, it was deduced that the bearings added to Model 3 reduced axial displacement for each rotational speed value by increasing stiffness and removing Model 3 based on the results.

Figure 5 shows the alternation in the torsional angle of experimental and numerical models by increasing rotational speed. It is observed that the torsional angle of all numerical models is lower than the experimental data. Nevertheless, the slope of curves in the figure are similar, indicating that the numerical models yield identical results despite the margin of error. Additionally, Model 1 performed very close results to the experiment. The maximum torsional angle for Model 2 and Model 3 does not match reveals that the coupled vibration for each rotational speed value impacts torsional vibration. By comparing Model 1 and Model 3, it was found that the bearings added to Model 3 reduced the torsional angle for each rotational speed value and removed Model 3 from the results of the experiment. Further, the bearings in Model 3 diminished the longitudinal vibrations, including the torsional angle, due to the coupling effect, unlike Model 1.

Figure 6 shows the coupled and uncoupled vibration forms are given for Model 1 at $100 \mathrm{rpm}$ shaft speed. Coupled and uncoupled vibration amplitudes for longitudinal vibration, as shown in Figure 6, are $2.45 \times 10^{-5} \mathrm{~m}$. In Figure 7, the coupled and uncoupled vibration amplitudes for torsional vibration are $5.23 \times 10^{-7} \mathrm{rad}$ and $3.35 \times 10^{-8} \mathrm{rad}$, according to the order. Due to the lack of the propeller in the system, the results

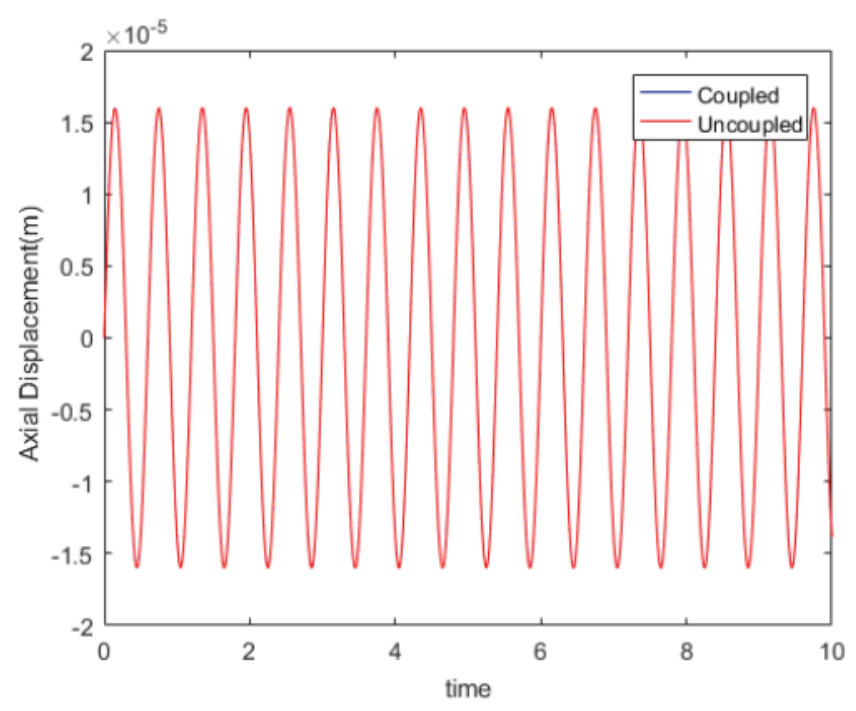

of hydrodynamic effects are not calculated. Thus, Model 2 is designed only for uncoupled vibrations, and Model 2 can only be compared with uncoupled vibration models. When examining differences between Model 2 with Model 1 , which is an uncoupled vibration model, the bearings in Model 2 only have an impact on axial displacement.

In Figure 7, the displacement values at $100 \mathrm{rpm}$ shaft speed are given for Model 3. When Model 1 compares the effect of coupled and uncoupled vibrations, the coupling effect on the torsional angle is highly efficient, but it has no impact on the axial displacement. Adding bearings to the system with Model 3 did not change this situation, and the coupling effect only affects the torsional angle in the system.

Figure 8 depicts the frequency of the force results from both experiment and numerical models for various rotational speed numbers. Since frequency values for longitudinal vibrations are tantamount to torsional vibrations, they are displayed in one graphic. The frequency of the external force is directly related to the speed of the shaft for numerical models and experiments. In order to detect the forcing frequency of the experiment and the margin of error, the transient response analysis was used. It was found that the margin of error is $8 \%$ for $100 \mathrm{rpm}$ and decreases until 160 rpm. However, thereafter it reincreased to $190 \mathrm{rpm}$. The validity of the experiment has also been controlled in this way.

\section{Conclusion and Comments}

Three numerical models are used to the dynamic behaviors of coupled torsional-longitudinal vibration for propulsion shaft system subjected to exciting torque and longitudinal force. The dynamic response of displacements and frequency response for the system with individual and

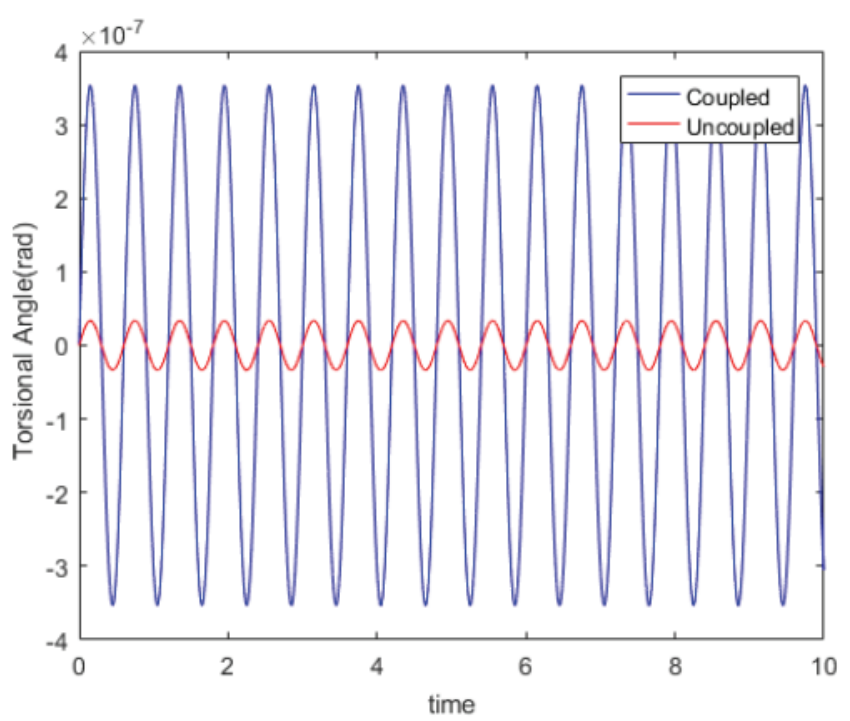

Figure 7. Axial displacements and torsional angles for Model 3 at $100 \mathrm{rpm}$ 


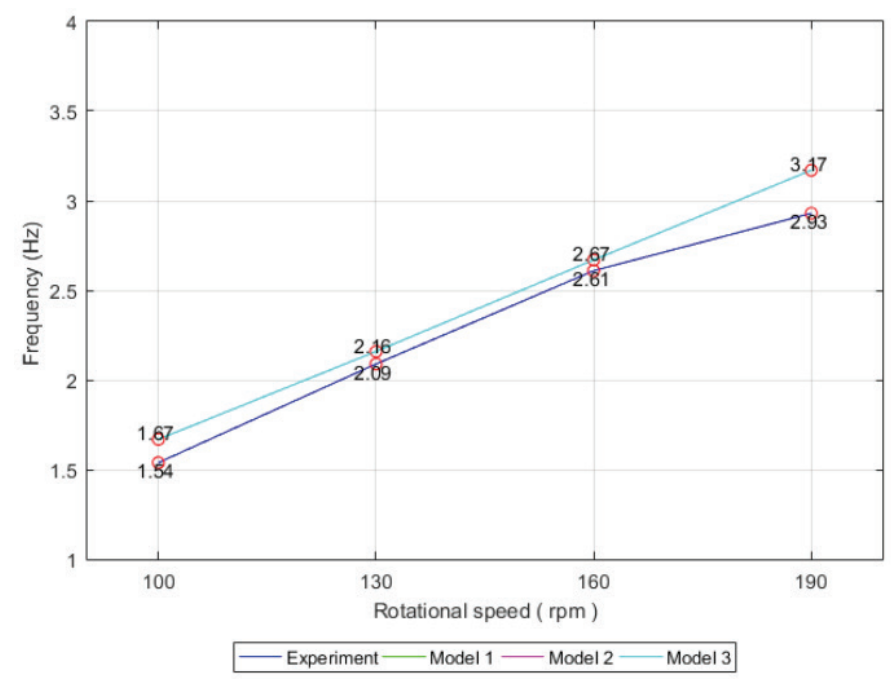

Figure 8. The driving frequency results at $\omega=100-190 \mathrm{rpm}$

coupled incentives are discussed in detail. Some conclusions are obtained as follows:

The coupling effect is not found during the axial displacement, but it is highly effective on the torsion angle. When the effect of the bearings on the system was considered, the axial displacement and torsion angle values decreased as stiffness increased, and the experimental results were removed. Likewise, it was inferred that the bearings reduced the axial displacement by increasing stiffness for uncoupled vibration and reduced the torsion angle by the coupling effect. This inference leads to obtaining the result that torsional and longitudinal vibration are mutually coupled with each other.

The propulsion shaft system was overly simplified and unrealistic when it was considered without bearings. In contrast, the inclusion of bearings in the numerical model increased the margin of error for the coupled torsion angle and longitudinal displacement. As a result, when the system was brought closer to reality by adding bearings to Model 1 , despite providing a fast solution for coupled torsionallongitudinal vibration problems, Model 1 was inconvenient and invalid in a realistic propulsion shaft system with an increased error rate.

\section{Acknowledgments}

This research is supported by the Scientific and Technological Research Council of Turkey (TUBITAK) 2214A International Doctoral Research Fellowship Programme. Experiments were performed at the Wuhan University of Technology. The authors offer sincere thanks to Professor Xinping Yan, Cong Zhang, and Qianwen Huang at Wuhan University of Technology for their valuable support and guidance during the corresponding author's academic visit to China. The corresponding author also offers sincere thanks to Asst. Prof. Bahadır Uğurlu and Ress. Ass. İsmail Kahraman from İstanbul Technical University for their support and patience.

\section{Authorship Contributions}

Concept design: A. N. Halilbeșe, O. A. Özsoysal, Data Collection or Processing: A. N. Halilbeșe, O. A. Özsoysal, Analysis or Interpretation: A. N. Halilbeșe, O. A. Özsoysal, Literature Review: A. N. Halilbeşe, O. A. Özsoysal, Writing, Reviewing and Editing: A. N. Halilbeșe, O. A. Özsoysal.

Funding: The author(s) received no financial support for the research, authorship, and/or publication of this article.

\section{References}

[1] I. Andersen, and J. Jensen, "Measurements in a container ship of wave-induced hull girder stresses in excess of design values," Marine Structures, vol. 37, pp. 54-85, Jul 2014.

[2] L. Murawski, and A. Charchalis, "Simplified method of torsional vibration calculation of marine power transmission system," Marine Structures, 39,335-349, Dec 2014.

[3] H., Han, K. Lee, and S. Park, "Estimate of the fatigue life of the propulsion shaft from torsional vibration measurement and the linear damage summation law in ships," Ocean Engineering, vol. 107, pp. 212-221, Oct 2015.

[4] Q. Huang, X. Yan, C. Zhang, and H. Zhu, “Coupled transverse and torsional vibrations of the marine propeller shaft with multiple impact factors," Ocean Engineering, vol. 178, pp. 48-58, April 2019.

[5] A. N. Halilbese, C. Zhang, and O. A., Özsoysal, "Effect of coupled torsional and transverse vibrations of the marine propulsion shaft system," Journal of Marine Science and Application, vol. 20, pp. 201-212, Jun 2021.

[6] L. Brydum, and S. B Jakobsen, "Vibration characteristics of two-stroke, low speed diesel engines," International Marine Propulsion Conference, 9th, MAN B\&W Diesel a/s, Copenhagen, pp. 1-16, London, 1987.

[7] Y. Huang, and C. Horng, "Analysis of torsional vibration systems by the extended transfer matrix method," Journal of Vibration and Acoustics, vol. 121, pp. 250-255, April 1999.

[8] J. S. Wu, and I. H. Yang, "Computer method for torsion-andflexure coupled forced vibration of shafting system with damping," Journal of Sound and Vibration, vol. 180, pp. 417-435, Feb 1995.

[9] Q. Huang, X. Yan, and Y. Wang, "Numerical and experimental analysis of coupled transverse and longitudinal vibration of a marine propulsion shaft," Journal of Mechanical Science and Technology, vol. 30, pp. 5405-5412, Dec 2016.

[10] L. Murawski, "Axial vibrations of a propulsion system taking into account the couplings and boundary conditions," Journal of Marine Science and Technology, vol. 9, pp. 171-181, Dec 2004.

[11] I. Senjanovića, I. Ančića, G. Magazinovićb, N. Alujevića, N. Vladimira, and D. Choc, "Validation of analytical methods for the estimation of the torsional vibrations of ship power transmission systems," Ocean Engineering, vol. 184, pp. 107-120, Jul 2019. 
[12] L. Xiang, S. Yang, and C. Gan, "Torsional vibration of a shafting system under electrical disturbances," Shock and Vibration, vol. 19, 1-11, 2012.

[13] D. Zou, J. Xu, J. Zhang, F. Lv, N. Ta, and Z. Rao, "The hydroelastic analysis of marine propellers considering the effect of the shaft: theory and experiment," Ocean Engineering, vol. 221, pp. 108547, Feb 2021.

[14] Z. Gan-boa, and Z. Yaoa, "Reduced-order modeling method for longitudinal vibration control of propulsion shafting," 2nd International Conference on Mechanical, Industrial, and Manufacturing Engineering, IERI Procedia, vol. 1, pp. 73-80, 2012.

[15] L. Dai, Z. Zhang, and D. Sun, "A study on axial, later and torsional coupled vibration of marine propulsion system with transfer matrices method, Shipbuilding of China, vol. 4, pp. 98-106, 1989. (in Chinese),"

[16] Q. Huang, X. Yan, Y. Wang, C. Zhang, and Z. Wang, "Numerical modeling and experimental analysis on coupled torsionallongitudinal vibrations of a ship's propeller shaft," Ocean Engineering, vol. 136, pp. 272-282, May 2017.

[17] M. Parsons, "Mode coupling in torsional and longitudinal shafting vibration," Marine Technology, vol. 20, pp. 257-271, Jul 1983.

[18] D. Zou, C. Jiao, N. Ta, and Z. Rao, "Forced vibrations of a marine propulsion shafting with geometrical nonlinearity (primary and internal resonances)," Mechanism and Machine Theory, vol. 105, pp. 304-319, Nov 2016.
[19] D. Zou, L. Liu, Z. Rao, and N. Ta, “Coupled longitudinal-transverse dynamics of a marine propulsion shafting under primary and internal resonances," Journal of Sound and Vibration, vol. 372, pp. 299-316, Jun 2016.

[20] S. Hassan, and G. Mehrdaad, "Free vibration of Timoshenko beam with finite mass rigid tip load and flexural-torsional coupling," International Journal of Mechanical Sciences, vol. 48, pp. 763-779, Jul 2006.

[21] Q. Huang, J. Liu, C. Zhang, and X. Yan, "Analysis on propulsion shafting coupled torsional-longitudinal vibration under different applied loads," Inter-noise, Conference paper Melbourne, Australia, 2014.

[22] D. Zou, Z. Rao, and N. Ta, "Coupled longitudinal-transverse dynamics of a marine propulsion shafting under super harmonic resonances," Journal of Sound and Vibration, vol. 346, pp. 248264, Jun 2015.

[23] Q. Huang, H. Liu, and J. Cao, "Investigation of lumped-mass method on coupled torsional-longitudinal vibrations for a marine propulsion shaft with impact factors," Journal of Marine Science and Engineering, vol. 7, pp. 95, April 2019.

[24] G. Kouroussis, and O. Verlinden, "Prediction of railway ground vibrations: accuracy of a coupled lumped mass model for representing the track/soil interaction," Soil Dynamics and Earthquake Engineering, vol. 69, pp. 220-226, Feb 2015.

[25] Z. Tian, "Study of vibration model for large scale ship shafting subjected to hull deformations and main engine excitations," Ph.D. dissertation, Wuhan University of Technology, China, 2016. 